\title{
CORRESPONDENCE
}

\section{Anti-tuberculosis drugs and liver toxicity}

\section{To the Editor:}

We read with great interest the Review Article entitled "Antituberculosis medication and the liver: dangers and recommendations in management" by THOMPSON et al. [1] published in the August 1995 issue of the Journal. We feel that the article is both highly informative and practically useful for respiratory physicians. However, we would like to present here an additional short commentary based on some local data and a supplementary review of the literature on this very important subject.

A small retrospective analysis of patients who developed hepatic dysfunction whilst on antituberculosis drugs, hospitalized in our Unit between 1st January 1991 and 31st December 1992, was recently undertaken. Out of 1,181 patients who received rifampicin, isoniazid with or without pyrazinamide and other drugs, 142 developed clinically symptomatic hepatic dysfunction. The biochemical findings included: 1) an elevation of serum alanine aminotransferase (ALT) level to $\geq 3$ times normal, with or without elevation of serum bilirubin level; or 2) an isolated persistent or progressive rise of serum bilirubin level to $\geq 2$ times normal. These 142 cases were treated as index patients. Another 234 patients who had regular liver function monitoring on a monthly basis, or even more frequently, up to 4 months after treatment commencement and who had not developed liver dysfunction were viewed as control patients. These two groups had comparable mean age and body weight. An assumption that the vast majority of hepatic dysfunction episodes should have occurred within 2 months of commencement of antituberculosis chemotherapy was made, as generally reported [2]. Indeed, 139 out of the 142 index patients developed hepatic dysfunction within 4 months of treatment in our analysis.

Eleven conditions were investigated for their potential as risk factors for antituberculosis drug-related hepatic dysfunction. These included: 1) heroin addiction; 2) long-term steroid administration; 3) heavy alcohol consumption $\left(>60 \mathrm{~g} \cdot \mathrm{day}^{-1}\right) ; 4$ ) hepatitis B carrier status (HBsAg positive); 5) diabetes mellitus; 6) elevated serum urea $\left.\left(>6.7 \mathrm{mmol} \cdot \mathrm{L}^{-1}\right) ; 7\right)$ elevated serum creatinine $(>120$ $\left.\left.\mu \mathrm{mol} \cdot \mathrm{L}^{-1}\right) ; 8\right)$ decreased serum albumin $\left.\left(<35 \mathrm{~g} \cdot \mathrm{L}^{-1}\right) ; 9\right)$ decreased lymphocyte count $\left.\left(<1.0 \times 10^{9} \cdot \mathrm{L}^{-1}\right) ; 10\right)$ elevated baseline ALT $\left(>38 \mathrm{IU} \cdot \mathrm{L}^{-1}\right)$; and 11$)$ elevated baseline bilirubin $\left(>17 \mu \mathrm{mol} \cdot \mathrm{L}^{-1}\right)$. Using statistical analysis, by cross-tabulation method (Chi-squared), it was found that amongst the 11 factors, the p-value was less than 0.01 for hepatitis B carrier status, heavy alcohol consumption and elevated baseline ALT only. Further analysis revealed that hepatitis B carrier status and alcohol consumption might be independent risk factors $(p=0.27)$, but elevated baseline ALT was probably related to hepatitis B carrier status $(\mathrm{p}<0.01)$ and heavy alcohol consumption $(\mathrm{p}<0.05)$. Furthermore, five patients were found to be seropositive for hepatitis $\mathrm{C}$ in the index group, but unfortunately not all patients were routinely tested for antibody to hepatitis C. Thus, in summary, these findings corroborate the message clearly depicted in the Review Article that those patients with underlying liver disease seem to be more prone to liver injury by antituberculosis drugs.

However, there are still a few caveats. Firstly, as indicated in some reports, the occurrence of hepatitis in a patient who has received antituberculosis drugs could represent coincident recent infection with hepatitis B or hepatitis A rather than true drug reaction [3-5]. This is exemplified by the presence of anti-hepatitis B core immunoglobulin $\mathrm{M}(\operatorname{IgM})$ and anti-hepatitis A virus IgM in some patients. The significance with hepatitis A is likely to be even greater than hepatitis B because it has no propensity to cause chronic liver derangement and, thus, would not pose any additional future risk of antituberculosis drug toxicity when the patient has recovered from the acute hepatitic episode. We recently encountered a young man who developed hepatitis during the fifth month of antituberculosis chemotherapy, whilst receiving rifampicin and isoniazid. He was subsequently found to be suffering from acute hepatitis A and was put back on isoniazid and rifampicin, with good tolerance when he had fully recovered from hepatitis A. Secondly, acute exacerbations of hepatitis in patients with chronic hepatitis B virus (HBV) infection can occur in chronic HBV carriers [6]. These episodes are associated with abrupt changes in the level of HBV replication, and reflect the host attempt to clear the virus or immune response against reactivated virus. These viral replicative changes in chronic HBV carriers can only be assessed by viral deoxyribonucleic acid (DNA) levels and cannot be easily differentiated from hepatic dysfunction induced by antituberculosis drugs. Thus, in communities where hepatitis B and hepatitis A (and perhaps hepatitis $\mathrm{C}$ as well) prevail, the above two possibilities should also be borne in mind.

Regarding the liver toxicity resulting from co-administration of rifampicin and isoniazid, it is also our experience that some fluoroquinolones are useful as component drugs in the interim or definitive treatment regimens in some patients in the face of hepatic dysfunction [7, 8]. Whilst, in some patients, withdrawal of pyrazinamide allowed them to be put back on treatment with both isoniazid and rifampicin, co-administration of isoniazid and rifampicin could not be tolerated in others. The latter group of patients had to receive isoniazid or rifampicin plus the fluoroquinolone and ethambutol only as 
definitive drug regimens. In our preliminary experience, there did not seem to be clear evidence that isoniazid proves much more injurious than rifampicin and, in this connection, we consider that it is the combination of these two drugs that confer the additive, or even synergistic, potential of liver toxicity than either agent alone, as conjectured [9-11].

\section{References}

1. Thompson NP, Caplin ME, Hamilton MI, et al. Antituberculosis medication and the liver: dangers and recommendations in management. Eur Respir J 1995; 8: $1384-1388$

2. Girling DJ. Adverse effects of antituberculosis drugs. Drugs 1982; 23: 56-61.

3. Kumar A, Misra PK, Mehotra R, Govil YC, Rana GS. Hepatotoxicity of rifampicin and isoniazid: is it all druginduced hepatitis? Am Rev Respir Dis 1991; 143: $1350-1352$.

4. Amarapurkar DN, Prabhudesai PP, Kalro RH, Desai HG. Antituberculosis drug-induced hepatitis and $\mathrm{HBsAg}$ carriers. Tubercle Lung Dis 1993; 74: 215-216.

5. Türktas H, Ünsal M, Tülek N, Örüc O. Hepatotoxicity of antituberculosis therapy (rifampicin, isoniazid) or viral hepatitis. Tubercle Lung Dis 1994; 75: 58-60.

6. Lok ASF, Lai CL. Acute exacerbations in Chinese patients with chronic hepatitis B virus (HBV) infection: incidence, predisposing factors and etiology. J Hepatology 1990; 10: 29-34.

7. Yew WW, Lee J, Wong PC, Kwan SYL. Tolerance of ofloxacin in the treatment of pulmonary tuberculosis in presence of hepatic dysfunction. Int J Clin Pharm Res 1992; XII: 173-178.

8. Yew WW, Chau CH, Wong PC, et al. Ciprofloxacin in the management of pulmonary tuberculosis in the face of hepatic dysfunction. Drugs Exptl Clin Res 1995; XXI: 79-83.

9. Yasuda K, Sato A, Chida K, et al. Pulmonary tuberculosis with chemotherapy related liver dysfunction. Kekkaku 1990; 65: 407-413.

10. $\mathrm{Wu} \mathrm{J}-\mathrm{W}$, Lee S-D, Yeh P-F, et al. Isoniazid-rifampicin induced hepatitis in hepatitis B carriers. Gastroenterology 1990; 98: 502-504.

11. Steele MA, Burk RF, Des Prez RM. Toxic hepatitis with isoniazid and rifampicin: a meta-analysis. Chest 1991; 99: 465-471.

W.W. Yew, C.H. Chau, S. Leung

Tuberculosis \& Chest Unit, Grantham Hospital, Hong Kong.

\section{REPLY}

\section{From the authors:}

YEW et al. report a $12 \%$ frequency of clinically symptomatic liver dysfunction during treatment with antituberculosis medication over a 12 month period. This is considerably higher than the $2.7 \%$ described in a metaanalysis [1], and may reflect the relative frequency of chronic liver disease due to viral hepatitis in Hong Kong. It is interesting that all those with symptomatic liver dysfunction had either a more than threefold rise in serum alanine transferase (ALT) or a persistent twofold rise in serum bilirubin; these were the biochemical parameters which we suggested as indicating significant hepatotoxicity. It should be noted that aspartate transferase (AST) is a more sensitive marker for significant alcoholic liver disease.

Although the great majority of patients developed hepatic dysfunction within 4 months, we note that it did occur at a later date in some patients $(n=3)$, and for this reason regular monitoring of liver function tests is required for the duration of antituberculosis therapy. As in earlier studies, Yew et al. showed that alcohol abuse [2] and chronic hepatitis B infection [3] appear to be independent risk factors for the development of liver dysfunction during treatment with antituberculosis medication. YEW et al. confirm the usefulness of fluoroquinolones as possible second-line agents, and also that rifampicin potentiates the hepatotoxicity of isoniazid $[4,5]$.

It is true that liver dysfunction in a patient taking antituberculosis medication may not be related to those drugs and may be due to concomitant liver disease. We note that only one case of concomitant hepatitis due to hepatitis A and no cases due to exacerbations of hepatitis B are quoted by YEw et al. As there is no specific treatment for acute viral hepatitis and "continuance of (drug) therapy once a hepatic reaction has commenced is the commonest cause of a fatal outcome" [6], we feel it would be prudent to assume that liver dysfunction is due to the antituberculosis medication, whilst a search for an alternative cause is sought if this is clinically appropriate. Even if the hepatitis is not due to the antituberculosis medication, it would be sensible to stop medication which is potentially hepatotoxic during an episode of acute, viral hepatitis - as was done by YEw et al.

\section{References}

1. Steele MA, Burk RF, DesPrez RM. Toxic hepatitis with isoniazid and rifampicin A meta-analysis. Chest 1991; 99: 465-471.

2. Gronhagen-Riska C, Hellstrom P-E, Froseth B. Predisposing factors in hepatitis induced by isoniazid-rifampicin treatment of tuberculosis. Am Rev Respir Dis 1978; 118: 461-466.

3. Chen GQ. The impact of antituberculosis drugs upon liver function in patients with positive HBVM. ChungHua Chieh Ho Ho Tsa Chih 1989; 12: 89-90.

4. Yasuda K, Sato A, Chida K, et al. Pulmonary tuberculosis with chemotherapy-related liver dysfunction. Kekkaku 1990; 65: 407-413.

5. $\mathrm{Wu} \mathrm{J}-\mathrm{W}$, Lee $\mathrm{S}-\mathrm{D}$, Yeh P-F, et al. Isoniazid/rifampicininduced hepatitis in hepatitis B carriers. Gastroenterology 1990; 98: 502-504.

6. Drugs and the liver. In: Sherlock S, Dooley J, eds. Diseases of the Liver and Biliary System. Oxford, Blackwell Scientific Publications, 1993; p. 350.

N.P. Thompson, M.E. Caplin, M.I. Hamilton, N. McIntyre, A.K. Burroughs

University Dept of Medicine, Royal Free Hospital School of Medicine, Rowland Hill St, London NW3 2PF. 